\title{
Veins based Authentication System
}

\author{
Amit P. Sahu \\ K.J Somaiya College of Engineering, Vidyavihar, \\ Maharashtra, India
}

\author{
Bharathi H.N \\ K.J Somaiya College of Engineering, Vidyavihar, \\ Maharashtra, India
}

\begin{abstract}
Biometric system such as finger, face, iris, ear, voice, DNA etc. is used for personal identification. Authentication based on vein pattern is an approach that uses the vast network of blood vessels that lie underneath a skin on back of the hand. Vein pattern are unique and also difficult to duplicate. In this paper, we present an approach to identify the user using hand vein pattern and knuckle shape extraction from hand vein image. Low cost IR camera imaging has been used to capture the image. The system consists of image acquisition, image pre -processing, feature extraction and matching. The score are generated in two stages for matching: minutiae feature extraction point technique from thinned vein pattern and the calculation of knuckle point perimeter distance from the acquired images. These two scores are used for matching, to authenticate the user.
\end{abstract}

\section{Keywords}

Minutaie, bifurcation, veins pattern, skeletionization, binarization

\section{INTRODUCTION}

Nowadays a very large number of identification systems exist that are based on different types of biometrics. This includes finger print, face, voice, DNA, signature, voice, hand geometry etc. Biometric recognition is based on fundamental premises about body traits: distinctiveness and permanence.

Fingerprints, face, and iris are amongst the most popular physiological characteristics used in commercial biometric systems. The following requirements that a given measure must satisfy to be a biometric: [1]

- Universality: each person should have the characteristic measured.

- Distinctiveness: any two persons should be sufficiently different in terms of this characteristic.

- Permanence: this characteristic should be sufficiently invariant (with respect to the matching criterion) over a period of time.

- Collectability: this characteristic should be measurable quantitatively.

Veins pattern can be defined as a random mesh of blood carrying tubes on back of the hand. These veins pattern are unique even in identical twins and universal. These are mainly two types of hand veins found on the dorsum of the hand namely cephalic and basilic. The basilic veins are group of veins attached with surface of hand and cephalic veins are attached with the elbow. The structure of the vein pattern can be acquired either through thermal imaging, also called far Infrared or through near infrared imaging. NIR is more viable option for building a cheap image capture setup. The dominant component of the skin is water, which absorbs the light with wavelength below $300 \mathrm{~nm}$ and above $1000 \mathrm{~nm}$. Two other component i.e. hemoglobin and melanin are the main absorbent of light in the visible spectrum (400-600 nm).From 650 to $1100 \mathrm{~nm}$, absorption from the main components of the blood deoxy and ox hemoglobin, dominates. Therefore when NIR is used, the vein absorbs more light than the surrounding tissues and hence veins become more visible. So this kind of imaging is suited to reveal the underlying pattern of the blood vessels.

\section{PRIOR WORK}

The uniqueness and the difficult duplication of hand vein pattern have attracted the attention of researcher for its usage in the personal identification. Lin and Fan has presented the personal identification using palm dorsal images acquired from the thermal infrared camera. Wang and Leedham uses NEC thermal tracer to acquire thermal images of the back of the hand. These images where captured at the normal office environment. It has been observed that the images are captured through a reflection based system as proposed [2]. Extraction of the region of interest (ROI) is the first step after image capture. The problem with ROI extraction is that the region extracted has to be of the same size and position for every picture taken. To resolve this, methods for extracting the ROI exist. Wang and Leedham [3], proposes to fix the hand at a certain position that is immediately below the camera. [5] This paper has proposed to crop the image to locate the ROI and perform the preprocessing operation on the cropped image. Cross and Smith applied thinning followed by a pruning algorithm to get rid of small unnecessary branches. [4] [5] many paper used Hausdroff distance for comparing the similarities between the images.

\section{PROPOSED SYSTEM}

In this paper, we have developed a veins based security system by utilizing the minutiae point and knuckle features [8]. The approach uses dome infrared camera and USB TV tuner to capture the back hand veins image. This paper can be summarized as follows:

(i) The extraction of bifurcation point and the ridge ending point from the skeleton image

(ii) Knuckle shape feature is extracted from the acquired binaries image. 
The block diagram shows the proposed approach in the fig.

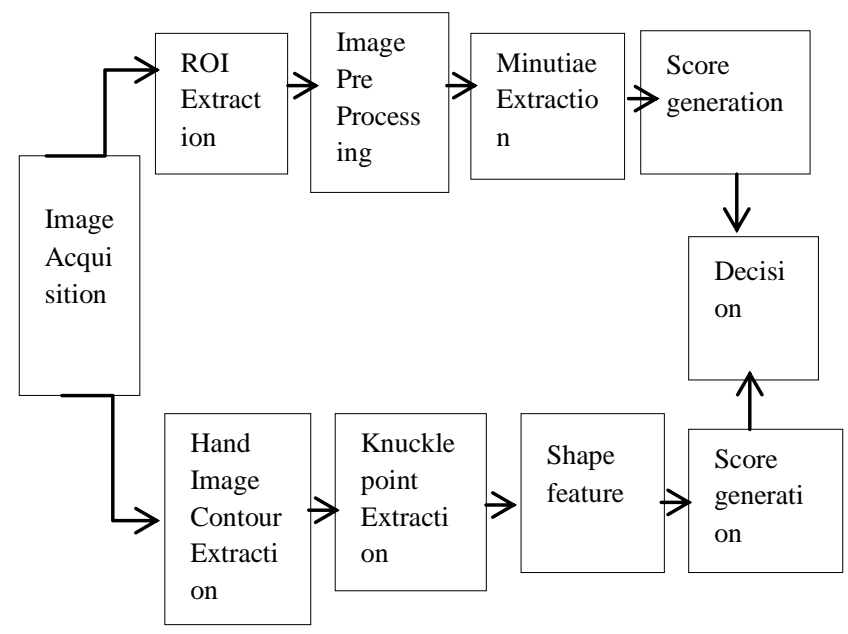

Fig 1. Flow diagram of proposed system

\subsection{Image Acquisition}

Near Infrared imaging technique is used for hand vein image acquisition. Low cost near IR dome camera is used to capture the veins at the back of the hand. The infrared LED is circularly mounted around the camera is position above the hand at the distance between the camera and hand is $18 \mathrm{~cm}$ for the acquisition. There are several ways to constraint the hand to prevent rotation and translation. A hand grip and rod is used where the user is requested to present their folded right hand. When hand is exposed to infrared light, then the deoxidized hemoglobin in the veins tube absorbs the light then the surrounding area and appears darker.The image is acquired using the setup is shown in fig.

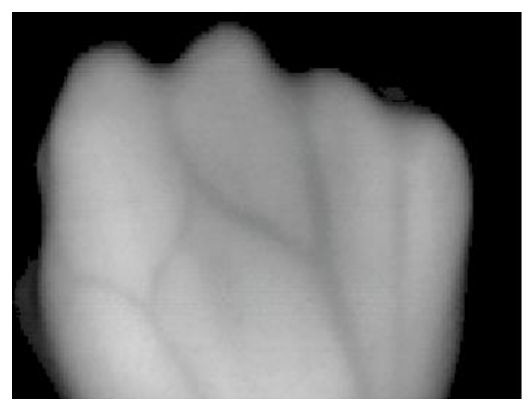

Fig. 2 Image acquired from the setup

\subsection{Image Pre-processing}

The image that is acquired through camera generally contains some unwanted information which is not required to obtain the veins pattern. The image captured from camera will be enhanced through several image preprocessing stages.

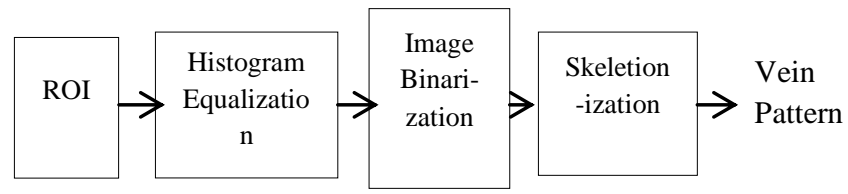

Fig. 3 Flow diagram of image preprocessing

\subsubsection{Knuckle point extraction}

In order to obtain the knuckle point from the acquired image canny edge detection algorithm is used as shown in the fig.

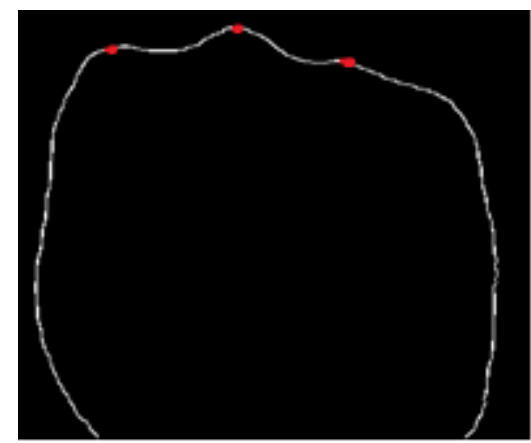

Fig. 4 Knuckle tips from hand contour

The knuckle tips can be extracted by scanning the contour image from left to right and from top to bottom. The point where the first pixel occurs from white to black is the first knuckle point as $(\mathrm{Tm})$. The second knuckle point $(\mathrm{Ti})$ and third point $\left(T_{r}\right)$ is obtained by scanning from left side and right side of $(\mathrm{Tm})$.

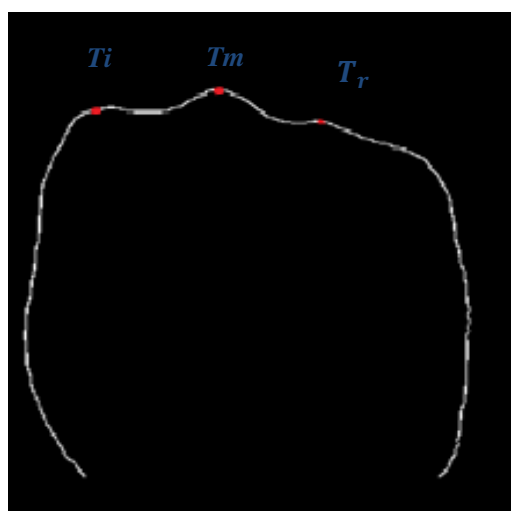

Fig. 5 Knuckle tips point $\left(T i, T m, T_{r}\right)$

In our implementation the point $(\mathrm{Tm})$ is approximated contour point between $(T i)$ and $\left(T_{r}\right)$.Once the key control points $(T i$, $T m, T_{r}$ ) are located, these pointes are used for further calculation as follows,

$$
\begin{aligned}
L e_{1} & =\sqrt{\left(T_{m}\right)^{2}-\left(T_{i}\right)^{2}} \\
L_{2} & =\sqrt{\left(T_{r}\right)^{2}-\left(T_{i}\right)^{2}}
\end{aligned}
$$

$L e_{1} \& L e_{2}$ is the point difference between $(T i,, T m)$ and $\left(T i, T_{r}\right)$.

The angle $\theta_{1}$ is calculated from point $\left(T i, T m, T_{r}\right)$ by calculating the slope $\mathrm{M}_{1}, \mathrm{M}_{2}$ as follows,

$$
\begin{gathered}
M_{l}=\frac{T_{m}(y)-T_{i}(y)}{T_{m}(x)-T_{m}(x)}-\cdots--(i) \\
M_{l}=\frac{T_{r}(y)-T_{i}(y)}{T_{r}(x)-T_{m}(x)} \text {------ }(i i)
\end{gathered}
$$


From (i) and (ii) angle $\theta_{1}$ is calculated i.e.

$$
\theta_{1}=\tan \left[\frac{M_{1-} M_{2}}{1-\left(M_{1}-M_{2}\right)}\right] \text {. ------- (iii) }
$$

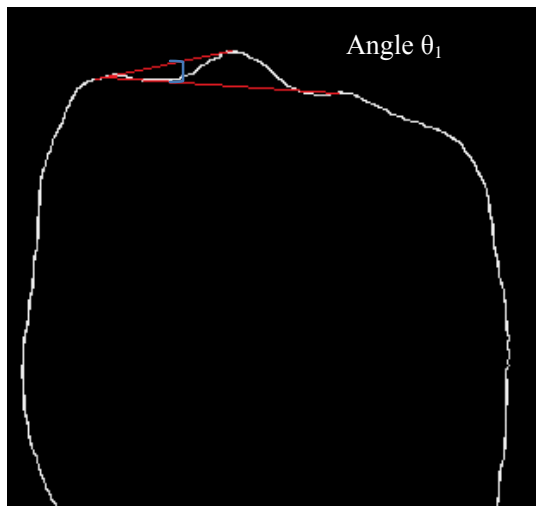

Fig. 6 Angle1 from the knuckle tip

Similarly angle $\theta_{2}$ is calculated by performing step (i), (ii), (iii) from points $(T r, T m, T i)$

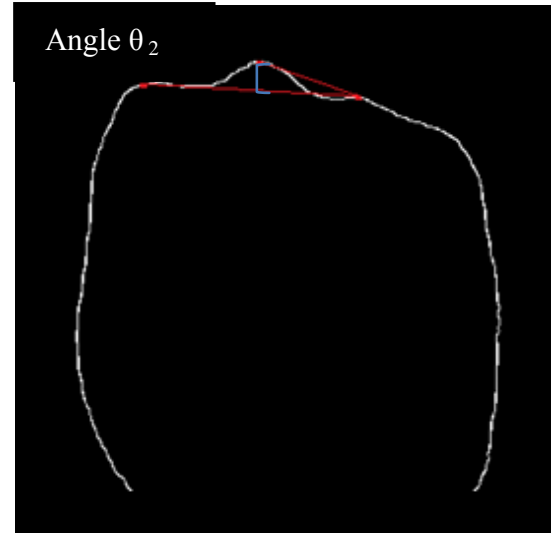

Fig. 7 Angle2 from the knuckle tip

\subsubsection{Extraction of vein pattern}

The Region of Interest (ROI) is obtained from the hand vein image for extraction of vein structure. The ROI is then obtained as a rectangle with upper left corner at pixel specified by the knuckle point $\left(T i, T_{r}\right)$. The resulting ROI extracted from the images are shown in figures

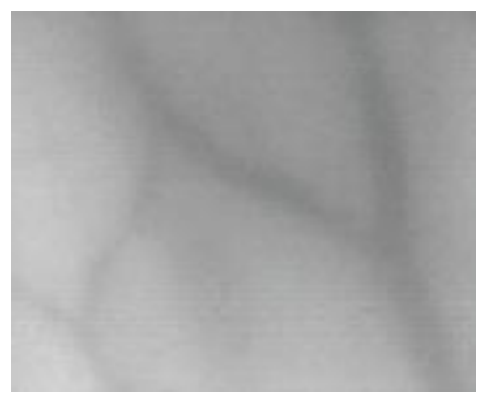

Fig. 8 Extracted ROI from the image

\subsubsection{Image Enhancement}

The image acquired from the camera is having a low quality in which the vein patterns are not clearly distinguish. To overcome this problem, contrast enhancement is applied to improve the contrast of vein image. A simple and very effective method to perform the contrast enhancement histogram equalization is used. Histogram equalization makes the dark pixel appear darker and light pixels appear lighter

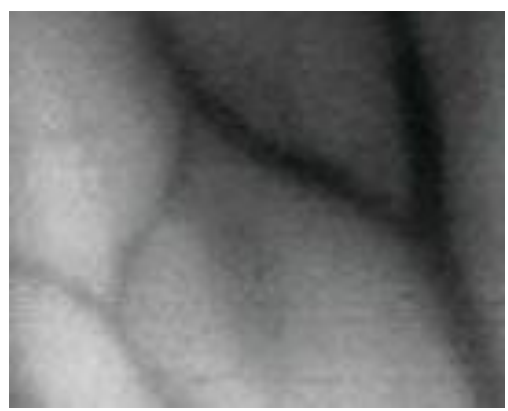

Fig. 9 Enhanced image

\subsubsection{Image binarization}

A binary image is an image that only uses two value for its pixel value $(0,1$ or 0 to 255$)$.To binarize an image, we will use local thresholding [7]. If $d(x, y)$ is the threshold version of an input image $f(x, y)$ with some threshold $\mathrm{T}$ then the thresholding process can be described as:

$$
d(x, y)=\left\{\begin{array}{l}
1 ; \text { if }(x, y) \geq T \\
0 ; \text { otherwise }
\end{array}\right.
$$

The proposed method describe in [7] is used for the binarization of the vein image.

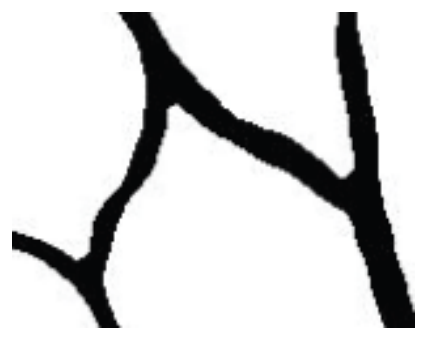

Fig 1. Flow diagram of proposed system

\subsubsection{Image skelentionzation}

Depending on different factors such as the ambient temperature, the diameter of veins can vary a lot even for one person. The global shape of the vein pattern needs to be extracted in order to perform an accurate recognition. To obtain a good representation of the shape of a vein pattern, the project focus on the extraction of what is called the skeleton of the vein pattern. The term skeleton describes the representation of a pattern by a collection of thin arcs and curves. Few methods exist to do so including skeletonisation and thinning [4]. The concept of them is to convert binary shapes to 1-pixel wide lines. Figure shows the results of the skeletonisation and the thinning algorithm. 


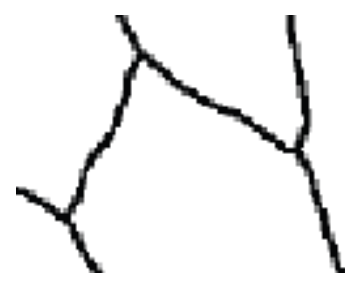

Fig. 11 Thinned image

\subsection{Feature Extraction}

In finger print biometric system the critical point is represented in finger pattern i.e. minutiae points. These minutiae points are used for matching the finger pattern. This technique can be used for the veins pattern in which vein bifurcation points are selected as the key points. A vein bifurcation is defined as vein points where vein forks or diverges into branch veins. To obtain the key points from the skeleton of vein pattern, we have used commonly known concepts of cross number [CN] [5]. Features like vein bifurcation are determined by computing the number of branches originating from the key pixel point. For a pixel P its eight neighbor pixels are scanned. For $3 \mathrm{X} 3$ region

\begin{tabular}{|c|c|c|}
\hline $\mathrm{P}_{1}$ & $\mathrm{P}_{2}$ & $\mathrm{P}_{3}$ \\
\hline $\mathrm{P}_{8}$ & $\mathrm{P}$ & $\mathrm{P}_{4}$ \\
\hline $\mathrm{P}_{7}$ & $\mathrm{P}_{6}$ & $\mathrm{P}_{5}$ \\
\hline
\end{tabular}

A given pixel $\mathrm{P}$ is termed as vein bifurcation point for a vein pattern, if the value of $N(P) \geq 3$. Mathematically this can be expressed as follows,

$$
N(P)=\sum_{i=1}^{8}\left|P_{i}+P_{i+1}\right|, \text { where } P_{9}=P_{1}
$$

The vein bifurcation pixel point is considered as key or feature points which can be defined as $(\mathrm{x}, \mathrm{y}, \theta)$ where $\mathrm{x}, \mathrm{y}$ are coordinate points of the pixel and $\theta$ is the orientation.

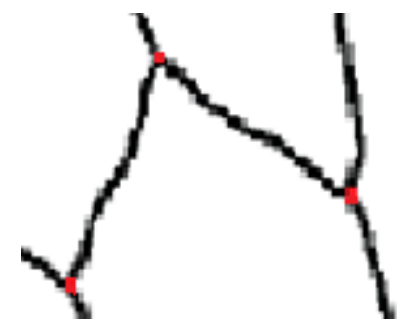

Fig. 12 Vein pattern with bifurcation point marked

\subsection{Matching}

Vein pattern matching is done by calculating the Euclidean distance between pair of veins pattern. Consider, $M \&$ M' are the two vein pattern for given pair of images.

$$
\begin{aligned}
& \left.M=\left\{\left(x_{1}, y_{1}, \theta_{1}\right),\left(x_{2}, y_{2}, \theta_{2}\right\}\right), \ldots .,\left(x_{i}, y_{i}, \theta_{i}\right)\right\} \\
& M^{\prime}=\left\{\left(x_{1}^{\prime}, y_{1}^{\prime}, \theta_{1}^{\prime}\right),\left(x_{2}^{\prime}, y_{1}^{\prime}, \theta_{2}^{\prime}\right), \ldots .,\left(x_{i}^{\prime}, y_{i}^{\prime}, \theta_{i}^{\prime}\right)\right\}
\end{aligned}
$$

Where $\left(x_{1}, y_{1}, \theta_{1}\right)$ and $\left(x_{1}^{\prime}, y_{1}^{\prime}, \theta_{1}^{\prime}\right)$ are the features key point from the vein pattern $\mathrm{M}$ and $\mathrm{M}^{\prime}$ respectively.

From a given key point the distance $D_{\mathrm{i}}$ and $D_{i}^{\theta}$ is calculated

$$
\begin{gathered}
D_{i}=\left|\sqrt{\left(x_{1}^{\prime}-x_{1}\right)^{2}+\left(y_{1}^{\prime}-y_{1}\right)^{2}}\right| \\
D_{i}^{\theta}=\left|\theta^{\prime}{ }_{1}-\theta_{1}\right|
\end{gathered}
$$

The vein pattern $M \& M^{\prime}$ are matched only if they satisfy following set of condition i.e.

$$
D_{i}<T_{i} \text { and } D_{i}^{\theta}<T_{\theta}
$$

Where $\mathrm{T}_{\mathrm{i}}$ and $T_{\theta}$ is the threshold

The score $S_{p}$ will be assigned for the maximum matching of key points.

The perimeter of pixels between the knuckle points ( $\mathrm{Ti}, \mathrm{Tm}$, $T_{r}$ ) from the normalized contour image is computed. The five features are used to perform separate knuckle shape feature i.e. Angles $\theta_{1}, \theta_{2}$ and the slope $M_{1}, M_{2}$ and the point difference. Euclidean distance is used for matching the two shape features. The score $S_{k}$ is assigned for the maximum matching of shape features.

$$
A=\left\{\begin{array}{l}
1 \text { if } S_{p}>t 1 \text { and } S_{k}>t 2 \\
0 \text { otherwise }
\end{array}\right.
$$

Where $\mathrm{t} 1$ and $\mathrm{t} 2$ is features threshold that is used for the calculation of $\mathrm{A}$ that is used for the decision making. If the value of $A$ is 1 , then one can judge the patterns are matched.

\section{CONCLUSION}

This system proposed an approach based on veins authentication using the minutiae feature technique and simultaneous extraction of knuckle point perimeter distance. This overall structure is composed by first image acquisition followed by preprocessing; binarization and finally feature extraction and recognition are performed. Image acquisition is done using cheap dome IR camera within the NIR wavelength. A setup has been built to normalize the images and constraint the hand movements (translation and rotation) in order to properly extract a fixed ROI. The image taken is then preprocessed using histogram stretching to enhance the image contrast. Among various binarization algorithms available for vein pattern recognition systems, the binary of the image was chosen to be done using local thresholding. Image thinning is performed on the binary image to obtain one pixel image of the vein pattern. Minutiae feature technique is used to extract the bifurcation point from the thinned veins pattern. Finally the matching is performed using the Euclidean distance method to validate the user.

Vein pattern technology is being considered into various authentication solutions for use in public places (access control, time and attendance, security, hospitals). This technology can be used to perform e-commerce transaction and M-banking services. 
In future work, the veins features extracted from the images can be used as feature code to generate one time password to perform online transaction. The user will be registered with veins pattern extracted from the veins image. The feature code will be generated based on the veins pattern extracted and this code will be used to perform various banking services.

\section{REFERENCES}

[1] A. K. Jain, P. Flynn, and A. A. Ross, Handbook of Biometrics. Springer, 2008, pp.253-270.

[2] L. Wang,C.G Leedham, "A Thermal Hand Vein Pattern Verification System" in Pattern Recognition and Image Analysis, of Lecture Notes in Computer Science, Springer 3687/pp. 58-65 (2005).

[3] L.Wang, G. Leedham, D. Siu-Yeung Cho, "Minutiae feature analysis for infrared hand vein pattern biometrics" in Pattern Recognition 41/3/pp.920-929 (2008).

[4] C.Y. Suen and T.Y. Zhang, "A Fast Parallel Algorithm for Thinning Digital Patterns". Communications of the ACM 27 (3). March 1984

[5] Madhuri M.Pal, Prof.R.W.Jasutkar, "Implementation of Hand Vein Structure Authentication Based System",
Proceedings of the IEEE International Conference on Communication Systems and Network Technologies, pp 115-116, 2012 .

[6] Gayathri S, K Gerard Joe Nigel,S Prabakar, "Low Cost Hand Vein Authentication System on Embedded Linux Platform”, IJITEE Volume-2 pp. 138-139, March 2013

[7] $\mathrm{Wu}$ Liusheng,Chen Jiaxin,Li Wei. Niblack-based Binaryzation Algorithm for Palm Vein Image . Communications Technology,2010, 43(1):112-114

[8] A. Kumar and K. V. Prathyusha, Personal authentication using hand vein trian- gulation and knuckle shape," IEEE Transactions on Image Processing, no. 38, pp.2127/2136, 2009

[9] Mohit Soni, Sandesh Gupta, M. S. Rao, Phalguni Gupta." A New Vein Pattern-based verification System", International Journal of Computer Science and Information Security, Vol. 8, No. 1, pp.58-63, 2010.

[10] FUJITSU.http://www.fujitsu.com/global/about/rd/20050 6palm-vein.html 